\title{
De lo presencial a lo virtual, un modelo para el uso de la formación en línea en tiempos de Covid-19
}

\section{Do presencial ao virtual, um modelo para o uso de formação online no tempo de Covid-19}

\section{From in-person to virtual class, a model for using online training in the time of Covid-19}

\author{
Roberto Canales Reyes ${ }^{1}$ \\ Juan Silva Quiróz ${ }^{2}$
}

\begin{abstract}
RESUMEN
La formación virtual ha ido lentamente ganando espacio en la Educación Superior, pero la emergencia del Covid-19 aceleró este proceso. Sin duda que los tiempos actuales son una gran oportunidad para innovar y para acercar la formación virtual a las comunidades educativas, pero es necesario hacerlo en forma apropiada, de lo contrario podemos obtener resultados no esperados y ante la mirada crítica de los estudiantes, invalidar esta modalidad de formación. Este artículo presenta un modelo de cómo enfrentar la formación virtual en tiempos de crisis, de cómo transitar de lo presencial a lo virtual, de modo de integrar las potencialidades que las tecnologías digitales nos proveen para generar entornos que promuevan aprendizajes de calidad en los estudiantes.
\end{abstract}

Palabras Claves: Formación virtual. E-learning. Docencia. Educación Superior. Aprendizaje en línea.

1 Universidad de Los Lagos. Osorno, Chile. E-mail: rcanales@ulagos.cl - https://orcid. org/0000-0002-1088-5004

2 Universidad de Santiago de Chile. Santiago, Chile. E-mail: juan.silva@usach.cl - https:// orcid.org/0000-0002-9817-402X 


\title{
RESUMO
}

A formação virtual vem ganhando espaço lentamente no Ensino Superior, mas o surgimento da Covid-19 acelerou esse processo. Sem dúvida, os tempos atuais são uma grande oportunidade para inovar e aproximar a formação virtual das comunidades educacionais, mas é necessário fazê-lo adequadamente, caso contrário, podemos obter resultados inesperados e, no olhar crítico dos alunos, invalidar essa modalidade de formação. Este artigo apresenta um modelo de como enfrentar a formação virtual em tempos de crise, de como passar do presencial para o virtual, integrando o potencial que as tecnologias digitais nos fornecem para gerar ambientes que promovam a aprendizagem de qualidade nos alunos.

Palavras-chave: Formação virtual. E-learning. Ensino. Ensino Superior. Aprendizado Online.

\begin{abstract}
Academic virtual training is slowly gaining ground in Higher Education, but the arrival of Covid-19 has accelerated this process. Undoubtedly, current times are a great opportunity to innovate and bring virtual training closer to educational communities, but it is necessary to do it properly, otherwise, the outcome can be unexpected and, in the critical view of students, it would invalidate this training modality. This article presents a model of how to face virtual training in times of crisis, how to move from a presencial classroom to a virtual one, integrating the potential that digital technologies provide us to generate environments that promote quality learning in students.
\end{abstract}

Keywords: Virtual training. E-learning. Teaching. Higher Education. Online Learning.

\section{Introducción}

En momentos de crisis, de aislamiento social y de confinamiento, pasar de la presencialidad en educación, a la virtualidad, ha significado un nuevo reto y desafío para la mayoría de las instituciones de educación superior. El desafío no es sólo desde el punto de vista del proceso de enseñanza y aprendizaje, sino también de las brechas a nivel de oportunidades de accesibilidad y tecnológicas que tienen los y las estudiantes para conectarse a la dinámica del proceso educativo. 
Algunas instituciones en forma incipiente estaban incorporando la modalidad b-learning, pues combina lo mejor de la presencialidad y virtualidad (MORTIS et al., 2015). La mayor presencia de la modalidad online se daba en la educación continua a través de diplomados, postítulos, cursos y Massive Open Online Course (MOOC).

Los roles de los actores del sistema educativo en este nuevo escenario cambian, adquiere mayor importancia el educando, debiendo los profesores con escasa experiencia en lo no presencial, adaptar recursos, materiales, contenidos y actividades tanto sincrónicas como asincrónicas, para no generar más desigualdades en los aprendizajes. Las habilidades de sus estudiantes a nivel de autorregulación, autoaprendizaje, planificación de los tiempos para estudiar y para desplegar procesos de metacognición son desafíos pendientes que aún persisten en la educación presencial, lo que conspira en la modalidad online para la formación crítica y reflexiva de los ciudadanos de la sociedad del conocimiento, temática que se evidencia sobre todo en tiempos de crisis.

En este nuevo contexto, no estamos suponiendo un uso de una plataforma de videoclases tipo Meet, Zoom, Jitsi Meet para simplemente dictar la clase, tampoco en una plataforma como Moodle, Backboard, Classroom, Elluminate usada como repositorio para subir materiales. Estamos impulsando un ambiente de aprendizaje mediado por las tecnologías que se expresa en un entorno virtual, donde confluyen las diversas tecnologías asociadas a los contenidos, recursos, materiales, actividades sincrónicas y asincrónicas, los sistemas de seguimiento, retroalimentación y evaluación.

\section{Las Tecnologías de la Información y Comunicación (TIC) en la docencia universitaria}

En distintos modelos educativos pedagógicos institucionales de la educación superior presencial se ha consagrado al estudiante como centro del aprendizaje, especialmente en los modelos socioconstructivistas o modelos basados en competencias. Sin embargo, al observar las prácticas educativas, se evidencia escenarios pedagógicos tradicionales, con escasez de repertorios que incorporen estrategias o metodologías activas y participativas (ADELL, 2004)

En este sentido, la situación de crisis actual, en la cual debemos prescindir de la presencialidad, adoptando el distanciamiento social para combatir la pandemia, se está obligado a realizar, en materia de docencia, un tránsito desde la educación presencial a la virtual, modificado modelos y referentes clásicos 
de realizar docencia en educación superior, cuyo control del proceso educativo de enseñanza y aprendizaje estaba en manos del docente, mediado por el programa curricular y sus guías de aprendizaje, contextualizando los escenarios pedagógicos en función de su disciplina.

Así, enseñar en entornos virtuales en educación superior ha significado transitar por distintas fases temporo-espaciales en términos técnicos y pedagógicos (GROS, 2018), relevando en una primera etapa el diseño y desarrollo de recursos multimedia, focalizados en la producción de contenidos orientados al proceso de aprendizaje del estudiante mediado por computador, para luego adicionar una etapa de uso masivo de internet y correo electrónico, lo que hizo pensar en el desarrollo de contenidos y juegos de aprendizaje en línea y entornos virtuales de aprendizaje o Content Management System (CMS), asociado a aulas y campus virtuales.

Luego, ha evolucionado en torno a la web 2.0 con énfasis en aplicaciones informáticas y móviles que potencian las relaciones sociales entre las personas, pasando de ser no sólo consumidores pasivos de información, sino que también usuarios activos, generadores de datos y contenidos. Actualmente, el foco está puesto además en la administración y gestión de la información y de datos en la nube y al desarrollo de contenidos y contextos educativos de aprendizaje libre, tales como los cursos masivos abiertos en línea Moocs.

Por otro lado, enseñar en entornos virtuales de aprendizaje en educación superior, asociado a dispositivos móviles, implica reconocer en el educando el desarrollo de un aprendizaje ubicuo, el cual trasciende al contexto temporo espacial de una clase tradicional, permitiendo al aprendiz potenciar competencias tecnológicas digitales y habilidades del siglo XXI, considerando las distintas fuentes y canales de información que lo nutren.

Así, los docentes universitarios al hacer uso de las tecnologías digitales en educación superior deben incorporar dentro de la modalidad virtual o eLearning métodos de enseñanza interactivos, con una relación articulada y directa entre el docente, estudiante y los materiales, considerando recursos y actividades pedagógicas que permiten trabajar el aprendizaje individualizado y autónomo, en función de los ritmos de aprendizaje de cada uno o una.

Las tecnologías digitales pueden potenciar el aprendizaje flexible y abierto asociado con la introducción de los medios sociales en la educación (SALINAS, 2013; MARÍN; NEGRE; PÉREZ-GARCÍAS, 2014). Para Selwyn (2013, p. 5). "Existe una aceptación generalizada de que las tecnologías digitales deben desempeñar un papel integral en la provisión de todos los aspectos del aprendizaje a lo largo de toda la vida, desde la integración de las computadoras en las aulas escolares y universitarias hasta la entrega virtual de cursos en línea y entrenamiento". 


\section{Rol del docente}

La tecnología ha ayudado en las últimas décadas a configurar nuevos espacios y modalidades en el ámbito de la educación y la cultura (CASTELLS, 2004). En la sociedad del conocimiento del siglo XXI es imperativo que quienes ejercen la docencia estén capacitados en conocimientos, habilidades y actitudes para el uso eficiente e inteligente de las TIC. Existen diversas iniciativas que han propuesto modelos, estándares e instrumentos de evaluación de la competencia TIC (MINEDUC-ENLACES, 2011; INTEF, 2017; UNESCO, 2011; FRASER; ATKINS; RICHARD, 2013). Aunque cada vez hay más estudios e investigaciones sobre esta competencia en enseñanza primaria y secundaria, las propuestas son menores si el contexto es el de la Educación Superior (MENGUAL; ROIG, 2012).

En la definición que realiza Gutiérrez Porlán (2014, p. 54) de competencia TIC en Educación Superior, destaca los aspectos principales que componen este término, definiéndola como: "los valores, creencias, conocimientos, capacidades y actitudes para utilizar adecuadamente las tecnologías, incluyendo tanto los ordenadores como los diferentes programas e Internet, que permiten y posibilitan la búsqueda, el acceso, la organización y la utilización de la información con el fin de construir conocimiento”. Prendes Espinosa \& Gutiérrez Porlán (2013), definen un marco de competencias TIC para la docencia en la Universidad Pública Española, el cual contempla las áreas: conocimiento general del ordenador y posibilidades de las TIC, conocimiento y uso de las estrategias metodológicas para el trabajo en red, posibilidades y limitaciones de las TIC en el proceso de enseñanza-aprendizaje, elección recursos TIC para el aula, conocimiento y uso de herramientas, publicación de material en la red; uso de las TIC para diferentes tareas docentes, formación docente e innovación con TIC. Estas áreas se presentan en tres niveles: dominio de las bases que fundamentan la acción con TIC, diseño, implementación y evaluación de acción con TIC y análisis y reflexión personal y colectiva de la acción llevada a cabo con TIC. En el contexto latinoamericano (LÁZARO; GISBERT; SILVA, 2018) definen 22 indicadores agrupados en las dimensiones: didáctica, curricular y metodológica, planificación, organización y gestión de espacios recursos tecnológicos digitales, relacional, ética y seguridad; personal y profesional.

Para Salinas, De Benito \& Lizana (2014) será necesario que los docentes se desenvuelvan en el mundo digital, en la creación y distribución de contenidos y recursos, en diferentes contextos y con diferentes dispositivos. En este contexto, la docencia en espacios virtuales se presenta como un gran desafío, 
el docente se formó y aprendió de maestros en la clase presencial, tiene escasa o nula experiencia en la formación virtual y lo que implica ser docente en estos espacios formativos.

En el éxito de una experiencia formativa virtual el rol del docente "tutor" es muy importante. Garrison y Anderson (2005) señalan que los roles del tutor pueden clasificarse en tres categorías principales: diseño y organización, facilitar el discurso y enseñanza directa. Estos autores hacen referencia a la presencia docente para indicar el rol del tutor, entendido este rol como quien diseña, facilita y orienta los procesos cognitivos y sociales, con el objetivo de obtener resultados educativos significativos tanto para el aprendiz como para el propio docente.

\section{Los estudiantes}

La European Commission (2016) define las competencias clave para el ciudadano del siglo XXI como un conjunto de conocimientos, habilidades y actitudes para participar en la sociedad y aprendizaje al largo de la vida. Entre estas competencias se encuentra la Competencia Digital (CD), definida como: "el uso seguro, crítico y responsable de las tecnologías digitales en el ámbito personal (aprendizaje), en el laboral y en el social" (EUROPEAN UNION, 2018, p. 4). El marco más utilizado en la actualidad es el definido por la Comisión Europea, quien publicó el informe sobre la competencia digital denominado DIGCOM 2.0: un marco para el desarrollo y la comprensión de la Developing and competencia digital en Europa (VUORIKARI et al., 2016). La propuesta considera cinco áreas: alfabetización en información y datos, comunicación y colaboración, creación de contenido y seguridad y resolución de problemas (VUORIKARI et al., 2016).

Unesco (2015), en el marco de acción para la educación del 2030, destaca el potencial de las tecnologías digitales (TD) y la importancia de la formación en competencias tecnológicas en los procesos de formación para el acceso al mercado laboral. La CD es relevante en el desarrollo de los estudiantes de educación superior para un mejor rendimiento en el ámbito académico, su desarrollo personal y profesional.

Los jóvenes viven en una cultura de la interacción y su paradigma comunicacional se basa en la interactividad al usar un medio instantáneo y personalizable como Internet (OBLINGER; OBLINGER, 2005). Los estudiantes universitarios que acceden a un uso intensivo y creativo de las herramientas de Web Social, suelen adaptarse mejor a ambientes de enseñanza creativos en vez de tradicionales (DUART et al., 2008). 
Existen instrumentos para medir la CD en los Universitarios como: INCOTIC-Grado, que es una herramienta diseñada para realizar una evaluación autodiagnóstica de la competencia digital de los estudiantes de primer curso de grado, para poder ajustar la planificación de la docencia de esta competencia cuando sea necesario (GONZÁLEZ; ESPUNY; GISBERT, 2011), fue generado en España. Existe una versión INCOTIC-LA adaptado a Latinoamérica y que se encuentra en etapa piloto aplicándose en universidades Latinoamericanas. Otro instrumento es el ACUTIC, elaborado para el estudio de las actitudes que tienen los estudiantes universitarios hacia las TIC (MIRETE RUIZ; GARCÍASÁNCHEZ; HERNÁNDEZ PINA, 2015).

\section{Enseñar en entornos virtuales}

Distintas son las causas que dan origen a la educación virtual. Entre ellas se puede mencionar la necesidad de compatibilizar las labores propias de la vida cotidiana de las personas jóvenes y adultas con los procesos formativos a través de las tecnologías, articulando la actividad laboral con el desarrollo profesional y en otros casos enfrentando las circunstancias de aislamiento y/o alejamiento territorial con los centros de formación, sin considerar las limitaciones de tiempo para acceder a los procesos formativos regulares. Adicional a lo anterior, existen causas vinculadas al ámbito educativo que distan en parte de la enseñanza presencial, tales como la personalización del aprendizaje, la flexibilidad en el acceso a los contenidos, actividades y sistemas de plataformas virtuales de aprendizaje Learning Management Systems - LMS (DURÁN, 2015).

La enseñanza virtual o enseñanza mediada por tecnologías, especialmente por entornos virtuales de aprendizaje, requiere de un diseño pedagógico distinto al de la enseñanza presencial, que atienda y potencie la autonomía e independencia de los estudiantes en el proceso de aprendizaje, la capacidad de autorregulación y de marcar los ritmos y tiempos en el aprendizaje. En este sentido especial cuidado se debe tener con los estudiantes con menos capacidades de autorregulación, en términos justamente de los tiempos dedicados al estudio, de la planificación y rutas de aprendizaje planteadas para el logro de objetivos de aprendizaje. Las evidencias investigativas indican (GROS, 2018) que los estudiantes más exitosos desde el punto de vista educativo y de rendimiento escolar en contextos de virtualidad, son aquellos que fueron más eficientes y eficaces en sus procesos de autorregulación. 
Pasar de lo presencial a lo virtual, implica desplegar buenas prácticas en entornos virtuales de aprendizaje, sobre todo considerando las exigencias de aislamiento y distancia social. Para ello la planificación de actividades curriculares deben ser significativas para los estudiantes, que inviten a la implicación del alumnado, al tratamiento a la diversidad y a la participación colaborativa y cooperativa de todos y todas.

\section{De la presencialidad a la virtualidad por la contingencia del COVID-19}

En Chile el e-Learning en el ámbito Universitario es incipiente, a pesar que las distancias geográficas y la concentración de las instituciones de educación superior se sitúan en las grandes ciudades. Existen pocas universidades que oferten e-Learning en pregrado, las experiencias se centran en cursos, diplomados y postgrados, con pocos casos a nivel de másters online, sólo 17 títulos y uno de ellos acreditado. Para incentivar la oferta de formación virtual en pregrado y postgrado, la Comisión Nacional de Acreditación de Chile (CNA) dispone de estándares para la acreditación de universidades, programas de pregrado y de postgrado (CNA, 2020).

En el escenario actual, la contingencia del COVID-19 ha forzado a las Universidades a recurrir a la formación virtual para poner en marcha el año académico, así observamos que se han adoptado diversas medidas:

- Uso de plataformas virtuales y herramientas de videoconferencia: Las universidades han optado por proveer de herramientas asíncronas como plataformas virtuales (Moodle, Canvas, Blackboard, etc.) y herramientas síncronas de videoclases como: Zoom (algunas han comprado la versión pro), Teams (del paquete de Microsoft), Meet de Google. Estas herramientas se complementan en la docencia en proporciones variadas, definidas normalmente por el cuerpo académico a nivel de departamentos o facultades al interior de las universidades. Las formas de trabajo son comunicadas a los estudiantes para que estos planifiquen su tiempo y dedicación al estudio.

- Elaboración de videotutoriales de apoyo para docentes. Estos funcionan como recursos de aprendizaje que enseñan el componente instrumental del uso de plataformas y herramientas de videoclases, pequeñas microcápsulas de creación de foros, envío de tareas, pruebas en línea, etc. 
- Elaboración de orientaciones generales para la docencia mediante entornos virtuales: cartillas pedagógicas o documentos que permiten situar la docencia en estos espacios formativos, fundamentando la modalidad y entregando principios orientadores con tips a tener presente en el diseño de los cursos, estructura, tiempos recomendables para realizar videoclases, sistemas de retroalimentación y evaluación.

- Call center de ayuda para el acceso y manejo de las plataformas: se han reforzado o creado estas instancias para resolver problemas técnicos asociados principalmente al acceso y manejo de herramientas de la plataforma y videoclases.

- Capacitaciones en el uso de herramientas de videoclases: sesiones de 1 a 2 horas para capacitar en el uso de las herramientas, especialmente de videoconferencia. En el caso de las plataformas se habían entregado talleres presenciales en el año y el verano.

- Webinar: seminarios en línea donde expertos en uso e integración curricular de las tecnologías digitales reflexionan en torno a los desafíos actuales.

- Planes para proveer de computador y/o acceso a internet: en Chile existe una brecha digital que impide que el 100\% de los estudiantes accedan a computador e Internet, en estos casos, las casas de estudio han implementado planes para dotar a los estudiantes más necesitados de dichas tecnologías.

Más allá de los esfuerzos realizados y las pertinencias de ellos dada la contingencia, se ha observado que no se ha realizado ninguna acción formativa relacionada con dos aspectos centrales de la docencia online (SILVA, 2011, p. 12): el diseño instruccional online y la tutoría. El primero busca que la docencia virtual que se está realizando no se transforme en una translado de la clase presencial al espacio virtual, la generación de repositorio de recursos, y en algunos casos al uso exclusivo de la videoclases como sustituto de la clase presencial centrada en el docente. El uso de la plataforma no debiera centrarse exclusivamente para la creación de foros y la entrega de tareas. Se requieren elementos de diseño instruccional online para el diseño de contenidos y actividades que integren las herramientas de la plataforma y las tecnologías digitales, bajo metodologías activas para situar el estudiante al centro del proceso de enseñanza aprendizaje. La tutoría es vital en el éxito de la formación virtual (SALMON, 2000; SILVA; ASTUDILLO, 2013; MARCELO, 2006), ella abarca la dimensión social para acoger al alumnado e integrarlo a una comunidad virtual, y en la dimensión pedagógica para orientar el proceso de aprendizaje, animar los espacios de comunicación para generar interacción reflexión y no solo participación. En síntesis, no ha existido una formación para que los docentes integren de forma efectiva la virtualidad a la docencia. 
La respuesta de los alumnos es variada y relacionada con la propuesta online que el docente despliegue, en los casos donde hay innovación, distribución de tiempos sincronos y asincronos adecuados, un diseño de actividades online que conjuguen lecturas y generación de productos y discusiones, la evaluación es positiva. En los casos contrarios, donde el modelo realiza la presencialidad de las clases en los mismos horarios asignados en el modelo presencial, la evaluación es negativa, los alumnos reportan cansancio y aburrimiento. El alumnado se cuestiona la modalidad especialmente cuando les demanda mucho tiempo o cuando no observan un cambio respecto a la docencia presencial. Un aspecto interesante observado por docentes y alumnos es que estos espacios ya sea síncronos o asíncronos permiten que se expresen alumnos que en la clase presencial no lo hacen, da la impresión que el medio digital al serles más cercanos favorece la participación. Llama la atención además, que los estudiantes cuando participan de la clase en línea, no encienden micrófonos ni cámaras de video, razones pueden ser varias, entre ellas conservar la privacidad desde sus hogares, realizar más de una actividad al mismo tiempo, entre otras.

El tema de las competencias digitales y en particular las competencias digitales docentes, surgen como factor importante. Existen docentes, especialmente de más edad o aquellos más alejados de las tecnologías que se les dificulta el uso de las mismas, lo cual los deja expuestos frente al alumnado y complejiza que hagan un buen uso de las tecnologías e integrarlas en forma natural en este espacio de formación virtual.

Silva et al. (2018), a propósito de las CDD, entregan recomendaciones para mejorar el uso didáctico, curricular y metodológico de las tecnologías y su impacto en el aula y en los aprendizajes, situación que es pertinente recordar en este contexto. Tabla 1

\section{TABLA 1 - DIMENSIÓN DIDÁCTICA, CURRICULAR Y METODOLÓGICA}

\begin{tabular}{|l|l|}
\hline \multicolumn{1}{|c|}{ Indicador } & \multicolumn{1}{c|}{ Recomendación } \\
\hline $\begin{array}{l}\text { Realiza búsquedas de } \\
\text { información accediendo } \\
\text { a diversas fuentes de } \\
\text { diversa tipología }\end{array}$ & $\begin{array}{l}\text { - Socializar estrategias de uso en el acceso a fuentes científicas. } \\
\text { - Integrar al currículo de los programas académicos de pedagogía a } \\
\text { nivel macro y micro curricular. } \\
\text { - Fomentar prácticas profesionales auténticas con uso de TIC. } \\
\text { - Estimular el análisis crítico de la información. }\end{array}$ \\
\hline $\begin{array}{l}\text { Utiliza software de apoyo } \\
\text { para la realización de } \\
\text { actividades de EA }\end{array}$ & $\begin{array}{l}\text { - Validar internamente los recursos tecnológicos en las comunidades } \\
\text { educativas. } \\
\text { - Integrar el diseño e implementación de actividades (por ejemplo, } \\
\text { selección de recursos tecnológicos) con uso de TIC en la asignatura } \\
\text { de didáctica. }\end{array}$ \\
\hline
\end{tabular}

Continúa 
REYES, R. C.; QUIRÓZ, J. S. De lo presencial a lo virtual, un modelo para el uso de la formación...

Conclusión
\begin{tabular}{|l|l|}
\multicolumn{1}{|c|}{ Indicador } & \multicolumn{1}{c|}{ Recomendación } \\
\hline $\begin{array}{l}\text { Diseña actividades de EA } \\
\text { donde contempla el uso } \\
\text { de tecnología digital }\end{array}$ & $\begin{array}{l}\text { - Socializar las transformaciones que implica realizar las TIC en } \\
\text { las estructuras didácticas de las distintas disciplinas pedagógicas. } \\
\text { - Diseñar e implementar situaciones didácticas y evaluar aprendizajes } \\
\text { de estudiantes, mediante actividades de integración de TIC. }\end{array}$ \\
\hline & $\begin{array}{l}\text { - Visibilizar las herramientas para la elaboración de instrumentos de } \\
\text { evaluación, potenciando el desarrollo de espacios de talleres o ferias } \\
\text { que ilustren experiencias de aprendizaje y procesos pedagógicos. } \\
\text { - Concientizar la transformación en los modelos o estilos evaluativos } \\
\text { que implica el uso de las TIC. }\end{array}$ \\
$\begin{array}{l}\text { Utiliza recursos digitales } \\
\text { para el seguimiento y } \\
\text { evaluación del alumnado }\end{array}$ & $\begin{array}{l}\text { que reconozcan las necesidades educativas para hacer mejores } \\
\text { mediaciones, dentro de una lógica de proceso. } \\
\text { - Utilizar los recursos de las plataformas de aprendizaje en línea } \\
\text { para hacer seguimiento al proceso de aprendizaje y evaluación de } \\
\text { producto. }\end{array}$ \\
\hline
\end{tabular}

FUENTE: SILVA et al., 2018.

Los docentes que dada su especialidad relacionada a las áreas de educación y tecnología y aquellos que habían tenido la experiencia de trabajar en programas virtuales, contaban con las competencias para diseñar y modelar cursos online, son quienes están salvando la situación en forma más eficiente. Así, la premura por virtualizar cursos que se realizaban en forma presencial ha implicado para los profesores una demanda de tiempo mayor para la docencia en condiciones de confinamiento, que no son las óptimas y que deben convivir con reuniones virtuales y tareas de investigación y gestión propias de la academia.

\section{El modelo}

La implementación de la docencia en entornos virtuales mediados por las tecnologías de la información y la comunicación requiere para su implementación efectiva, la articulación de las tecnologías digitales, recursos y actores y procesos educativos. Presentamos una propuesta (Figura 1) que muestra un modelo para producir una articulación virtuosa de los diferentes componentes de la docencia online mediada por tecnología, de tal forma que esto permita crear entornos de aprendizaje virtuales, que favorezcan aprendizajes de calidad. 
FIGURA 1 - MODELO DE DOCENCIA ONLINE

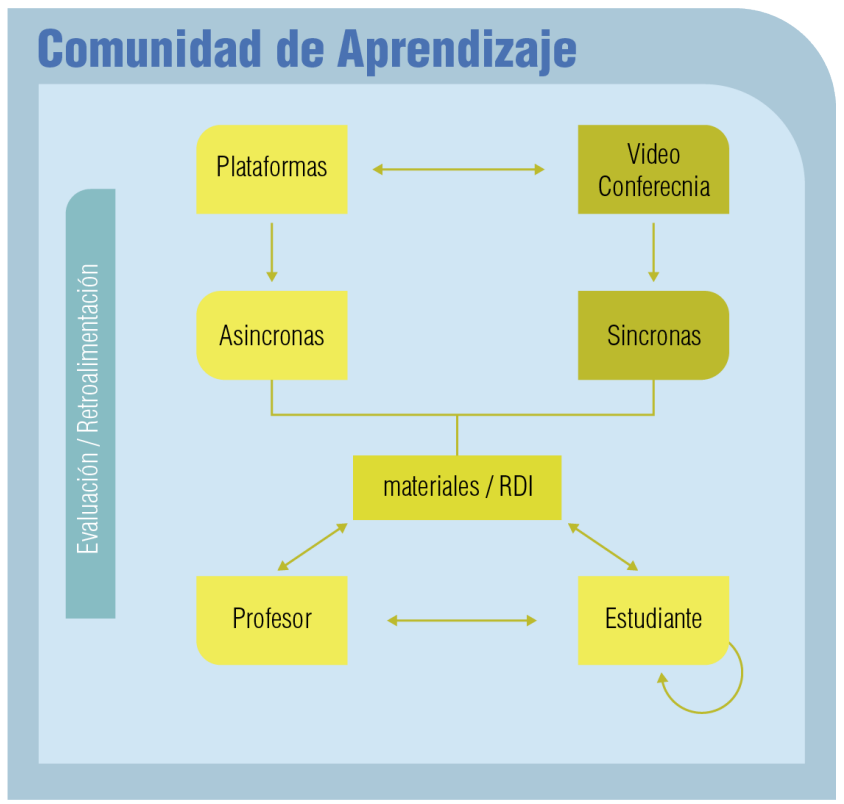

FUENTE: Elaboración propia.

A la base del modelo esta la comunidad de aprendizaje, concebida como un espacio para la construcción social de conocimiento. La comunidad de aprendizaje online bien implementada y guiada podría generar entornos educativos superiores al curso presencial Downes (2012). Una de las claves en estas comunidades es el conectivismo que las TIC facilitan, permitiendo colaborar y compartir con el objetivo final que es generar aprendizajes (Downes, 2013). Garrison y Anderson (2005) proponen que la comunidad online debe considerar la presencia social, cognitiva y docente.

En el modelo se presentan como apoyo dos tipos de Tecnologías Digitales (TD) básicas: un LMS para la comunicación asíncrona y plataformas de videoconferencia para la comunicación síncrona. El soporte tecnológico del modelo es la plataforma virtual o LMS (Moodle, Canvas, Dokeos, etc.), un espacio para diseñar ambientes de aprendizaje mediados por las TIC. En el modelo se contempla la modalidad e-Learning o $100 \%$ online, es decir sin instancias presenciales. La plataforma, considera las herramientas propias del LMS para desplegar contenidos, la colaboración, interacción, generación de productos, envíos de trabajo y evaluación. Así como las herramientas para 
acompañar el rol del profesor. Plataformas de Videoconferencia (Zoom, Meet, Teams, BigBlueButtonBN, Meet-Jitsi) que permiten el encuentro síncrono, incorporándose en algunos casos al LMS.

Se espera que las actividades tanto síncronas como asíncronas utilicen metodologías activas, tipo clase invertida (flipped classroom), estudio de casos, debates, basada en proyectos, juegos de roles, para así salir de la clase magistral y poner al estudiante al centro del proceso de aprendizaje y ofrecer nuevos escenarios formativos. El aprendizaje centrado en el estudiante considera sus características, conocimientos previos y estilos de aprendizaje.

La evaluación y retroalimentación son los aspectos más consultados por parte de los docentes al enfrentarse a la educación virtual. Para esto las orientaciones son claras: primero que todo especificar las rutas de aprendizaje dentro del escenario pedagógico o diseño instruccional, identificar los resultados de aprendizaje, considerar la estructuración y secuenciación de los contenidos a trabajar en distintas unidades temáticas, especificar bien las tareas y productos solicitados en los tiempos necesarios para desarrollar las actividades individuales o asociativas potenciando la interacción entre pares para la producción de resultados evaluativos de proceso o de productos, utilizando instrumentos tipo desarrollo de e-portafolios, ensayos, reportes de lectura, participación reflexiva en debates, foros de discusión, cuestionarios en línea, encuestas, glosarios, entrega de maquetas, prototipos, proyectos, entre otros.

Así, resulta clave la retroalimentación y seguimiento continuo del proceso de aprendizaje personal y por equipos de trabajo, considerando que la retroalimentación oportuna evita la deserción por el estudio, mejora la motivación y actitud frente al aprendizaje, dejando en evidencia la eventual sobrecarga cognitiva depositada en el estudiantes, debiendo desplegar estrategias de coordinación entre docentes que trabajan en los distintos cursos y niveles en el semestre lectivo para planificar en conjunto y en forma articulada los procesos evaluativos.

Normalmente en educación a distancia, los estudiantes van cursando un curso o temática a la vez. En cambio en este formato de tránsito de lo presencial a lo virtual, los estudiantes trabajan cursos en forma simultánea, cinco o seis asignaturas en línea, con docentes distintos, por tanto, es altamente relevante la coordinación de los equipos de trabajo.

El sistema se completa con tres aspectos básicos en todo modelo educativo actual: el profesor, los recursos y los estudiantes, considerando la interacción entre ellos. El profesor entendido como un facilitador juega un rol fundamental para acompañar el proceso formativo especialmente desde una dimensión pedagógica y social, velando por el correcto trabajo de los alumnos en las actividades y en constante interacción con el estudiante para guiar y orientar el 
trabajo. Materiales /recursos digitales contemplan los contenidos en forma de material escrito, presentaciones u otros formatos de autoría del profesor, videos, artículos, página web, blog, simulaciones, software, etc. disponibles en la web. Además de recursos digitales externos a la plataforma como herramientas para generar mapas conceptuales, infografías, lluvias de ideas, blogs, documentos colaborativos, entre otrasel aprendizaje del estudiante comienza en forma individual para luego fortalecerse en el aprendizaje en grupo con otros estudiantes. Esto permite al estudiante aprender no tan solo de los recursos y del profesor, sino también de los compañeros, en un escenario de construcción social del conocimiento.

\section{Recomendaciones para implementar el modelo}

Capacitación centrada en aspectos metodológicos que permitan aprovechar el potencial de las tecnologías digitales. En particular conocer el potencial de la plataforma que se use y las herramientas de videoconferencia. Estas formaciones deberían mezclar aspectos teóricos, metodológicos y prácticos, incentivar el promover metodologías activas centradas en los estudiantes y las características de las herramientas utilizadas en instancias formativas síncrona y asíncrona.

Formación en el rol del tutor, la docencia online requiere de competencias diferentes al de la docencia presencial, mencionaremos cinco aspectos: el diseño y la planificación de experiencias formativas virtuales centradas en las actividades más que en los contenidos; metodologías para innovar en espacios virtuales que consideren instancias sincronas y asincronas; la generación de recursos digitales (videos, presentaciones cápsulas, textos, etc.); la docencia online; el aprendizaje socioconstructivista que da espacio para una presencia social del estudiante a nivel personal y cognitivo.

Fomento del acceso de los docentes a espacios virtuales de aprendizaje. La crisis mundial marcó un antes y un después en la vida de todos y todas, en el caso de la educación superior muchos docentes se abrieron a la posibilidad de aprovechar las potencialidades de las tecnologías digitales, plataformas virtuales y sus recursos. Los estudiantes, sus aprendizajes, el trabajo individual y colaborativo se ven beneficiados, en términos de la innovación en la docencia.

Fomento de las habilidades y competencias digitales del siglo XXI es clave: trabajar con los estudiantes las habilidades de búsqueda de información, comunicación y colaboración, convivencia digital y habilidades tecnológicas. Además de las estrategias de aprendizaje autorreguladas, el autoaprendizaje, la metaevaluación y la metacognición. 
Fortalecimiento de las infraestructuras tecnológicas y acceso a la red, mejorando servidores, puntos de red inalámbricos, tecnología y soporte en las salas de clases, sistemas de gestión y administración de recursos tecnológicos en apoyo a la docencia. Incorporar software y recursos educativos open source y mejorando el acceso expedito a bases de datos científicas Web of Science WOS, Scopus, EBSCO, etc.

Apoyo Técnico vías mesa de ayuda que permitan resolver las consultas de los estudiantes respecto al acceso a la plataforma y el uso de ella para responder a las actividades sugeridas por el docente. En el caso de los docentes, una ayuda para auxiliarlos en el uso de las plataformas Content Management System (CMS) o herramientas de videoconferencia, de modo de resolver los problemas técnicos a los que se enfrentan.

Cursos bien diseñados, con contenidos interactivos, con material de apoyo y actividades que permitan el intercambio y colaboración entre estudiantes, contar con una batería de materiales en formato digital; manuales, guías, tutoriales, cápsulas, etc. que facilite al docente el uso de las plataformas online y herramientas de videoconferencia. Estos materiales deberían complementar el uso instrumental y pedagógico de las tecnologías que se usarán en la docencia.

Fomentar en los estudiantes el desarrollo de la Competencia Digital y en el futuro incorporarlo al perfil de egreso. Esto implica diagnosticar la CD y desarrollar acciones formativas para alcanzar su logro en la vida académica. En este contexto exponer a los estudiantes a experiencias formativas virtuales en modalidad e-learning, b-learning, MOOC u otra, será de vital importancia para desarrollar estas competencias y estar mejor preparados para futuras experiencias formativas virtuales y para el aprendizaje a lo largo de la vida.

Más allá de la circunstancias del COVID-19 y la docencia en espacios virtuales, es necesario que las instituciones definan un marco para entender la CDD en Educación Superior, al cual se inscribirá la institución, y generará instancias para evaluarlas, diseñar un plan formativo y para que los docentes se certifiquen y se mantengan actualizados.

\section{Conclusiones}

El uso de tecnologías digitales puede encontrar en los estudiantes un terreno fértil, dado el uso medios digitales que éstos emplean en su vida diaria. La investigación de Nowell (2014), informó que el uso de los medios sociales con estudiantes estadounidenses puede mejorar el potencial para el aprendizaje 
flexible y abierto asociado con la introducción de los medios sociales en la educación. Esto abre un potencial campo para incorporar innovaciones en la forma de concebir y llevar a cabo la docencia, que deberían tener una buena acogida en el estudiantado.

Es relevante que la experiencia formativa online sea un aporte al proceso formativo de los estudiantes, que perciban la innovación y la integración a la docencia al mundo digital en que ellos se desempeñan fuera del aula presencial. $\mathrm{Si}$ esta experiencia es frustrante, se corre el riesgo de generar un desencanto del estudiantado frente a la formación virtual que podría repercutir en el futuro en resistencias ante la modalidad para su formación permanente o continua.

Se recomienda que la entrega de contenidos sea por medios tecnológicos digitales asincrónicos, de tal manera que en las sesiones de sincronía a través de videoconferencia por ejemplo, se aprovechen para desarrollar la participación activa de los estudiantes a través de preguntas y respuestas, favoreciendo la interacción entre pares y con los docentes y tutores, de esta forma fomentar la socialización del aprendizaje colaborativo y cooperativo.

El desafío para la docencia universitaria que ha implicado el COVID-19 y la necesidad de pasar de una docencia presencial a la virtual nos da la posibilidad de realizar esfuerzos por unir el mundo digital en el que viven los estudiantes fuera del aula con la docencia, de modo de encantar a estos jóvenes y aprovechar al máximo su creatividad y potencialidad. Se requiere que las instituciones de educación superior particularmente sus docentes, hagan un uso innovador e inteligente de las tecnologías digitales. En este contexto las metodologías activas deberían asomar como las que iluminen las docencias, ellas se revitalizan con el uso de las tecnologías digitales, con las posibilidades del trabajo síncrono y asíncrono. Señalan (SALINAS; PÉREZ; DE BENITO, 2008) que no se crean metodologías nuevas, más bien las ya existentes se potencian con el trabajo colaborativo, el aprendizaje basado en proyectos, aprendizaje basado en casos, aprendizaje y servicio, etc. aunque hay otras metodologías como la clase invertida que nace al amparo de las tecnologías. Lo anterior a partir de las posibilidades que ofrecen las TIC de interacción, colaboración y ampliar la clase más allá de las fronteras del tiempo y la distancia. 


\section{REFERENCIAS}

ADELL, Jordi. Nuevas tecnologías en la formación presencial: del curso online a las comunidades de aprendizaje, Qurriculum: Revista de teoría, investigación y práctica educativa, España, n. 17, p. 57-92, 2004. Disponible en: https:/elbonia.cent.uji.es/jordi/ wp-content/uploads/docs/Qurriculum_2004.pdf. Acceso en: 13 abr. 2020.

CASTELLS, Manuel. The network society. A cross-cultural perspective. Massachusetts: Edward Elgar, 2004.

CNA (COMISIÓN NACIONAL DE ACREDITACIÓN). Santiago,Chile: CNA, 2020. Disponible en: https://bit.ly/2RHdylK. Acceso en: 13 abr. 2020.

DOWNES, Stephen. The Role of Open Educational Resources in Personal Learning. In: MCGREAL, Rory; KINUTHIA, Wanjira; MARSHALL, Stewart (ed.). Open Educational Resources: Innovation, Research and Practice. Vancouver, CA: Commonwealth of Learning, 2013. p. 203-226. Disponible en: https://goo.gl/cdv8Lg. Acceso en: 13 abr. 2020 .

DOWNES, Stephen. Connectivism and connective knowledge: Essays on meaning and learning networks. Canada: National Research Council Canada, 2012. Disponible en: https://www.downes.ca/files/books/Connective_Knowledge-19May2012.pdf. Acceso en: 16 abr. 2020.

DUART, Josep Maria et al. La Universidad en la sociedad RED, usos de la internet en educación superior. Barcelona: Ariel, 2008.

DURÁN, Rodrigo; ESTAY-NICULCAR, Christian; ÁLVAREZ, Humberto. Adopción de buenas prácticas en la educación virtual en la educación superior. Aula Abierta, Oviedo, n. 43, p. 77-86, 2015. Disponible en: https://www.sciencedirect.com/science/article/pii/ S0210277315000037?via\%3Dihub. Acceso en: 23 abr. 2020.

EUROPEAN UNION . Council Recommendation of 22 May 2018 on key competences for lifelong learning. Official Journal of the European Union, Brussels, v. 61, p. 189/1-189/13, june 2018. Disponible en: https://eur-lex.europa.eu/legal-content/EN/ TXT/?uri=OJ:C:2018:189:TOC. Acceso en: 23 abr. 2020.

FRASER, Josie; ATKINS, Lucy; RICHARD, Hall. DigiLit Leicester. Supporting teachers, promoting digital literacy, transforming learning. Leicester: Leicester City Council, 2013. Disponible en: https://bit.ly/2LDzSMw. Acceso en: 23 abr. 2020.

GARRISON, D. Randy; ANDERSON, Terry. El e-learning en el siglo XXI: investigación y práctica. Traducción de Alicia Fuentes Calle. Barcelona: Octaedro, 2005.

GROS, Salvat Begoña. La evolución del e-learning: del aula virtual a la red. RIED: Revista Iberoamericana de Educación a Distancia, Madrid, v. 21, n. 2, p. 69-82, 2018. Disponible en: http://revistas.uned.es/index.php/ried/article/view/20577/18099. Acceso en: 27 abr. 2020. 
GONZÁLEZ, Juan; ESPUNY, Cinta; GISBERT, Merce. INCOTIC. Una herramienta para la autoevaluación diagnóstica de la competencia digital en la universidad. Revista currículum y formación del profesorado, Granada, v. 15, n. 1, p. 75-90, 2011. Disponible en: http://www.ugr.es/ recfpro/rev151ART5.pdf. Acceso en: 13 abr. 2020.

GUTIÉRREZ PÓRLAN, Isabel. Perfil del profesor universitario español en torno a las competencias en tecnologías de la información y la comunicación. Pixel-Bit Revista de Medios y Educación, Sevilla, España, n. 44, p. 51-65, 2014. DOI: https://doi. org/10.12795/pixelbit.2014.i44.04. Acceso en: 13 abr. 2020.

INTEF (ed.). Marco Común de Competencia Digital Docente. Madrid: Ministerio de Educación, Cultura y Deporte, 2017. Disponible en: https://bit.ly/1Y88rd6. Acceso en: 27 abr. 2020.

LÁZARO, José Luis; GISBERT, Merce; SILVA, Juan Eusebio. Una rúbrica para evaluar la competencia digital del profesor universitario en el contexto latinoamericano. Edutec, Revista Electrónica de Tecnología Educativa, España, n. 63, p. 1-14, 2018. Disponible en: http://doi.org/10.21556/edutec.2018.63.1091. Acceso en: 27 abr. 2020.

MARÍN, Victoria; NEGRE, Francisca; PÉREZ-GARCIAS, Adolfina. Entornos y redes personales de aprendizaje (plepln) para el aprendizaje colaborativo [Construction of the Foundations of the ple and pln for Collaborative learning]. Comunicar, España, v. 21, n. 42, p. 35-43, 2014. Disponible en: https://doi.org/10.3916/C42-2014-03. Acceso en: 23 abr. 2020.

MARCELO, Carlos. Las nuevas competencias en E-learning ¿qué formación necesitan los profesionales del E-learning? En: MARTÍNEZ, Javier et al. Practicas de E-learning, Granada: Octaedro, 2006. p. 22-45.

MENGUAL, Andres; ROIG, Rosabel. La enseñanza y las competencias tic en el contexto universitario. En: Las tecnologías de la información en contextos educativos: nuevos escenarios de aprendizaje. Santiago de Cali: USC, 2012.

MINEDUC-ENLACES (ed.). Actualización de competencias y estándares TIC en la profesión docente. Santiago: Ministerio de Educación, Gobierno de Chile, 2011. Disponible en: https://bit.ly/2Q0zmqm. Acceso en: 23 abr. 2020.

MIRETE RUIZ, Ana Belén; GARCÍA-SÁNCHEZ, Francisco Alberto; HERÁNDEZ PINA, Fuensenta. Cuestionario para el estudio de la actitud, el conocimiento y el uso de TIC (ACUTIC) en Educación Superior. Estudio de fiabilidad y validez. Revista Interuniversitaria de Formación del Profesorado, Zaragoza, España, v. 29, n. 2, p. 75-89, 2015.

MORTIS, Sonia Verònica et al. La modalidad mixta: un estudio sobre los significados de los estudiantes universitarios. Innovación Educativa, México, DF, v. 15, n. 68, p. 73-97, 2015. Disponible en: http://www.scielo.org.mx/pdf/ie/v15n68/v15n68a6.pdf. Acceso en: 27 abr. 2020. 
NOWELL, Shanedra D. Using disruptive technologies to make digital Connections: stories of Media use and digital literacy in secondary Classrooms. Educational Media International, [s. l.], v. 51, n. 2, p. 109-123, 2014. Disponible en: https://doi.org/10.108 0/09523987.2014.924661. Acceso en: 27 abr. 2020.

OBLINGER, Diana G.; OBLINGER, James L. (ed.). Educating the Net Generation. Washington, D.C.: EDUCAUSE, 2005. Disponible en: https:/www.educause.edu/ir/ library/PDF/pub7101.pdf. Acceso en: 29 abr. 2020.

PRENDES ESPINOSA, Ma . Paz; GUTIÉRREZ PÓRLAN, Isabel. Competencias tecnológicas del profesorado en las universidades españolas. Revista de Educación, La Rioja, n. 361, p. 196-222, 2013.

SALINAS, Jesus. Enseñanza flexible y aprendizaje abierto, fundamentos clave de los ples. En: LINDA Castañeda; Jordi Adell (ed.). Entornos personales de aprendizaje: Claves para el ecosistema educativo en Red. Alcoy: Marfil, 2013. p. 53-70.

SALINAS, Jesús; DE BENITO, Barbara; LIZANA, Alexandra. Competencias docentes para los nuevos escenarios de aprendizaje. Revista Interuniversitaria de Formación del Profesorado, Zaragoza, España, v. 28, n. 1, p. 145-163, 2014.

SALINAS, Jesús; PÉREZ, Adolfina; DE BENITO, Barbara. Metodologías centradas en el alumno para el aprendizaje en red. Madrid: Síntesis, 2008.

SALMON, Gilly. E-moderating: The key to teaching and learning. New York; London: Routledge Taylor \& Francis Group, 2000.

SELWYN, Neil. Education in a Digital World: Global Perspectives on Technology and Education. London: Routledge, 2013.

SILVA, Juan; ASTUDILLO, Andrea. Formación de tutores aspecto clave en la enseñanza virtual.Didasc@lia: Didáctica y Educación, Cuba, v. 4, n. 1, p. 87-100, 2013.

SILVA, Juan et al. El desarrollo de la competencia digital docente durante la formación del profesorado. Opción, Zulia, v. 34, n. 86, p. 423-449, 2018.

SILVA, Juan. Diseño y moderación de Entornos Virtuales de Aprendizaje. Barcelona, España: Editorial UOC, 2011.

UNESCO. ICT competency framework for teachers. Paris: United Nations Educational, Scientific and Cultural Organization, 2011. Disponible en: https://bit.ly/2WD5kLH. Acceso en: 29 abr. 2020.

UNESCO. Educación 2030: Declaración de Incheon y Marco de Acción para la realización del Objetivo de Desarrollo Sostenible 4. En: FORO MUNDIAL SOBRE LA EDUCACIÓN 2015, Icheon, República de Corea: ONU, 2015. Disponible en: https:/www.gcedclearinghouse.org/sites/default/files/resources/245656s.pdf. Acceso en: 27 abr. 2020. 
REYES, R. C.; QUIRÓZ, J. S. De lo presencial a lo virtual, un modelo para el uso de la formación...

VUORIKARI, Riina et al. DigComp 2.0: The Digital Competence Framework for Citizens. Update Phase 1: The Conceptual Reference Model. Luxembourg: Publication Office of the European Union, 2016. Disponible en: https://publications.jrc.ec.europa. eu/repository/bitstream/JRC101254/jrc101254_digcomp\%202.0\%20the\%20digital\%20 competence $\% 20$ framework $\% 20$ for $\% 20$ citizens.\%20update\%20phase $\% 201$.pdf. Acceso en: 27 abr. 2020.

Texto recibido el 28/07/2020.

Texto aprobado el 11/09/2020. 